\title{
Rare Copy Number Variants Identify Novel Genes in Sporadic Total Anomalous Pulmonary Vein Connection
}

\begin{abstract}
Xin Shi't, Liangping Cheng ${ }^{1+}$, XianTing Jiao', Bo Chen ${ }^{1}$, Zixiong $\mathrm{Li}^{2}$, Yulai Liang ${ }^{3}$,

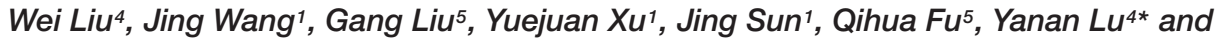
Sun Chen ${ }^{\text {* }}$

${ }^{1}$ Department of Pediatric Cardiovascular, Xinhua Hospital, School of Medicine, Shanghai Jiao Tong University, Shanghai, China, ${ }^{2}$ Department of Medical Oncology, Bayi Hospital, Nanjing University of Chinese Medicine, Nanjing, China, ${ }^{3}$ Institute of Health Sciences, Shanghai Institutes for Biological Sciences, Chinese Academy of Sciences, Shanghai, China, ${ }^{4}$ Department of Cardiothoracic Surgery, Xinhua Hospital, School of Medicine, Shanghai Jiao Tong University, Shanghai, China, ${ }^{5}$ Medical Laboratory, Shanghai Children's Medical Center, School of Medicine, Shanghai Jiao Tong University, Shanghai, China
\end{abstract}

OPEN ACCESS

Edited by:

Tao Huang,

Shanghai Institutes for Biological Sciences (CAS), China

Reviewed by:

Yujun Shen,

Tianjin Medical University, China

Daizhan Zhou,

Shanghai Jiao Tong University, China

*Correspondence:

Yanan Lu

luscmc@189.cn

Sun Chen

chensun@xinhuamed.com.cn

${ }^{t}$ These authors share first authorship

Specialty section:

This article was submitted to Bioinformatics and Computational

Biology,

a section of the journal

Frontiers in Genetics

Received: 29 August 2018 Accepted: 02 November 2018

Published: 23 November 2018

Citation:

Shi $X$, Cheng $L$, Jiao $X$, Chen $B$, Li Z, Liang Y, Liu W, Wang J, Liu G, Xu Y, Sun J, Fu Q, Lu Y and Chen S (2018) Rare Copy Number Variants Identify Novel Genes in Sporadic Total

Anomalous Pulmonary Vein

Connection. Front. Genet. 9:559.

doi: 10.3389/fgene.2018.00559
Total anomalous pulmonary venous connection (TAPVC) is a rare congenital heart anomaly. Several genes have been associated TAPVC but the mechanisms remain elusive. To search novel CNVs and candidate genes, we screened a cohort of 78 TAPVC cases and 100 healthy controls for rare copy number variants (CNVs) using whole exome sequencing (WES). Then we identified pathogenic CNVs by statistical comparisons between case and control groups. After that, we identified altogether eight pathogenic CNVs of seven candidate genes (PCSK7, RRP7A, SERHL, TARP, TTN, SERHL2, and $N B P F 3)$. All these seven genes have not been described previously to be related to TAPVC. After network analysis of these candidate genes and 27 known pathogenic genes derived from the literature and publicly database, PCSK 7 and TTN were the most important genes for TAPVC than other genes. Our study provides novel candidate genes potentially related to this rare congenital birth defect $(\mathrm{CHD})$ which should be further fundamentally researched and discloses the possible molecular pathogenesis of TAPVC.

Keywords: congenital heart defects, total anomalous pulmonary venous connection, whole-exome sequencing, copy number variants, pathogenesis

\section{INTRODUCTION}

Total anomalous pulmonary venous connection (TAPVC) is a rare but heterogeneous congenital heart anomaly in which pulmonary veins do not connect routinely to the left atrium but abnormally connect to the right atrium or systemic venous system. The incidence of TAPVC is approximately 1 out of 15,000 live births (Ammash et al., 1997; Bjornard et al., 2013; Thummar et al., 2014). TAPVC is rare but without proper intervention in the first year of life the mortality of TAPVC is nearly $80 \%$ (Burroughs and Edwards, 1960). However, the molecular mechanism of TAPVC remains unknown.

So far, only a few genes have been demonstrated as pathogenic genes for TAPVC and these genes are just a partial explanation for some patients. Bleyl et al. (2006) used genetic linkage analysis found a locus for TAPVR at 4p13-q12 called total anomalous pulmonary venous return 1 (TAPVR1) and other important pathogenic genes in this region include vascular endothelial growth factor 
receptor 2 (VEGFR2) and platelet-derived growth factor receptor $\alpha$ (PDGFRA). Nash et al. (2015) used whole genome sequence to identify a non-synonymous variant in the retinol binding protein 5 ( $R B P 5)$ gene which probably related to TAPVC. Li et al. (2017) considered activin A receptor type II-like 1 (ACVRL1) and sarcoglycan delta (SGCD) as TAPVC pathogenic genes using whole exome sequence from 6 TAPVC cases. However, these pathogenic genes explain only a small fraction of the molecular mechanism of TAPVC, the underlying cause in most patients remains unknown.

Copy number variant (CNV) is defined as a segment of DNA at least $1 \mathrm{~kb}$ in size that differs in copy number compared with a representative reference genome (Wellcome Trust Case Control Consortium et al., 2010; Pinto et al., 2011). CNVs have been shown to play an important role in the pathogenicity of complex birth defects (Greenway et al., 2009). CNV, or submicroscopic chromosomal deletions or duplications, has emerged as an important contributor to congenital genetic disorders and has identified critical dosage sensitive genes important for cardiac development (Hitz et al., 2012; Southard et al., 2012; Mlynarski et al., 2015). Whether CNV detection could be as a genetic selection for novel pathogenesis genes of TAPVC is still not reported previously, and it needs to be further studied.

\section{MATERIALS AND METHODS}

\section{Patient Ascertainment}

Our study recruited patients with TAPVC Xinhua Hospital Affiliated to Shanghai Jiao Tong University School of Medicine whose diagnoses were confirmed by echocardiography, cardiac catheterization, computed tomography, and other medical recordings. Patients with multiple major developmental anomalies, developmental syndromes, or major cytogenetic abnormalities were excluded. Ethical approval was given by the medical ethics committee of Xinhua Hospital.

\section{Detection of CNVs From WES Data}

Peripheral blood samples were obtained and DNA was extracted using the QIAamp DNA Blood Midi Kit (Qiagen, Germany). WES samples were captured with the Agilent Sure Select Target Enrichment kit (V6 58 Mb; Agilent Technologies, United States) and sequenced on the Illumina HiSeq 2500 platform (Glessner et al., 2014; Li et al., 2015). CNV coordinates were converted to the GRCh37/hg19 build using the UCSC Genome Browser LiftOver tool. CNVs with $50 \%$ or larger overlap with telomere, centromere, segmental duplications, or immunoglobulin regions were excluded (Hanemaaijer et al., 2012). After filtering we screened out rare CNVs by comparing with the Database of Genomic Variants $\left(\mathrm{DGV}^{1}\right)$ and Online Mendelian Inheritance in Man $\left(\mathrm{OMIM}^{2}\right)$.

\footnotetext{
${ }^{1}$ http://dgv.tcag.ca/
}

${ }^{2}$ http://omim.org

\section{Identification of Pathogenic CNV Candidates}

The CNV regions were firstly annotated with RefSeq genes. For each gene and each sample, the copy number status was determined by the annotated CNV regions. The pathogenic $\mathrm{CNV}$ candidates were then identified by statistical comparisons between the case and control groups. The statistical comparisons between groups were analyzed by one-side Fisher's exact test with alternative hypothesis that the mutation frequency is greater in case group than the control group. The $\mathrm{CNV}$ candidates were defined as potentially pathogenic if the $P<0.01$. The analysis and visualization were implemented in $\mathrm{R}$ programming software with version 3.5.0.

\section{Protein-Protein Interaction (PPI) Analysis}

Protein-Protein Interactions (PPI) are physical contacts with molecular associations between chains that occur in a cell or in a living organism in a specific biomolecular context (De Las Rivas and Fontanillo, 2010). Our candidate pathogenic genes with CNVs, combined with 27 known disease-causing genes derived from the literature and publicly available database, were mapped to PPI network in STRING database ${ }^{3}$ (Brohee et al., 2008), which identified the connections between the candidate pathogenic genes and the known disease-causing genes. Information found in STRING databases supports the construction of interaction networks (McDowall et al., 2009).

\section{Expression Patterns of the Selected Genes During Human Embryonic Heart Development}

Expression patterns of the human embryonic heart of candidate genes were detected using an Affymetrix HTA 2.0 microarray. To determine whether these candidate genes could affect human embryonic heart development, Carnegie stages 11 through 15 of human embryonic heart samples were collected from Xinhua hospital. RNA extraction used TissueLyser II (Qiagen, Germany) and the RNeasy MinElute Cleanup Kit (Qiagen, Germany) as previous study (Nolan et al., 2006). The integrity and purity of the RNA was detected by the Experion automated gel electrophoresis system (Bio-Rad, United States) and the NanoDrop 2000c spectrophotometer (Thermo Fisher Scientific, United States).

\section{RESULTS}

\section{Clinical Data}

A total of 78 sporadic TAPVC cases and 100 healthy controls were recruited in our research. Among these patients, no one had central nervous system malformations, vertebral defects, or genitourinary malformations. The patients' ages ranged from 27 days to 7 years; 45 patients were male $(57.7 \%)$ and 33 were female (42.3\%). Among all these patients, 47 had atrial septal defect (ASD), 16 had patent foramen ovale (PFO), 10 had ventricular septal defect (VSD), and 16 had patent ductus

${ }^{3} \mathrm{https}: / /$ string-db.org/ 
arteriosus (PDA). Double outlet right ventricle (DORV) was discovered in three patients, atrioventricular septal defect in three patients. The detailed clinical data and cardiac phenotypes are summarized in Table 1. All patients were recruited via Xinhua Hospital Affiliated to Shanghai Jiao Tong University School of Medicine and all signed an informed consent approved by the Ethics Committee of Xinhua Hospital.

TABLE 1 | The summary of 78 TAPVC patients.

\begin{tabular}{lc}
\hline Patient characteristics & Discovery cohort \\
\hline Mean age at diagnosis (years) & $0.95 \pm 1.87$ \\
BMl $\left(\mathrm{kg} / \mathrm{m}^{2}\right)$ & $14.92 \pm 3.05$ \\
Male $(n, \%)$ & $45(57.7)$ \\
Mortality $(n, \%)$ & $4(5.1)$ \\
Associated cardiac lesion $(\boldsymbol{n}, \%)$ & \\
ASD & $57(73.7)$ \\
PFO & $16(20.5)$ \\
VSD & $10(12.8)$ \\
PDA & $16(20.5)$ \\
DORV & $3(3.8)$ \\
AVSD & $3(3.8)$
\end{tabular}

\section{CNVs in Patients With TAPVC and Identification of Candidate Genes}

To discover the pathogenic CNV candidates, we identified WES data by statistical comparisons between the case and control groups. We use circos plot for CNV visualization with broad horizontal area from chromosome level (Figure 1). In all chromosomes, chromosome 1 had the most CNV numbers than other in our patients.

Based on these data, we identified statistically significant CNVs at different genomic loci. CNVs were filtered as potentially pathogenic if the $P<0.01$ (Table 2). Finally, we identified eight potentially pathogenic CNVs of seven genes (PCSK7, RRP7A, SERHL, TARP, TTN, SERHL2, and NBPF3) among 45 patients with TAPVC (Figure 2). The percentage of subjects with CNVs candidates was $58.4 \%$ (45 of 78 TAPVC subjects).

\section{Expression Pattern in Human Embryonic Heart}

We then detected the time course expression patterns of the candidate genes during different Carnegie stages of human heart development using microarray (Table 3). Expression of TNN in

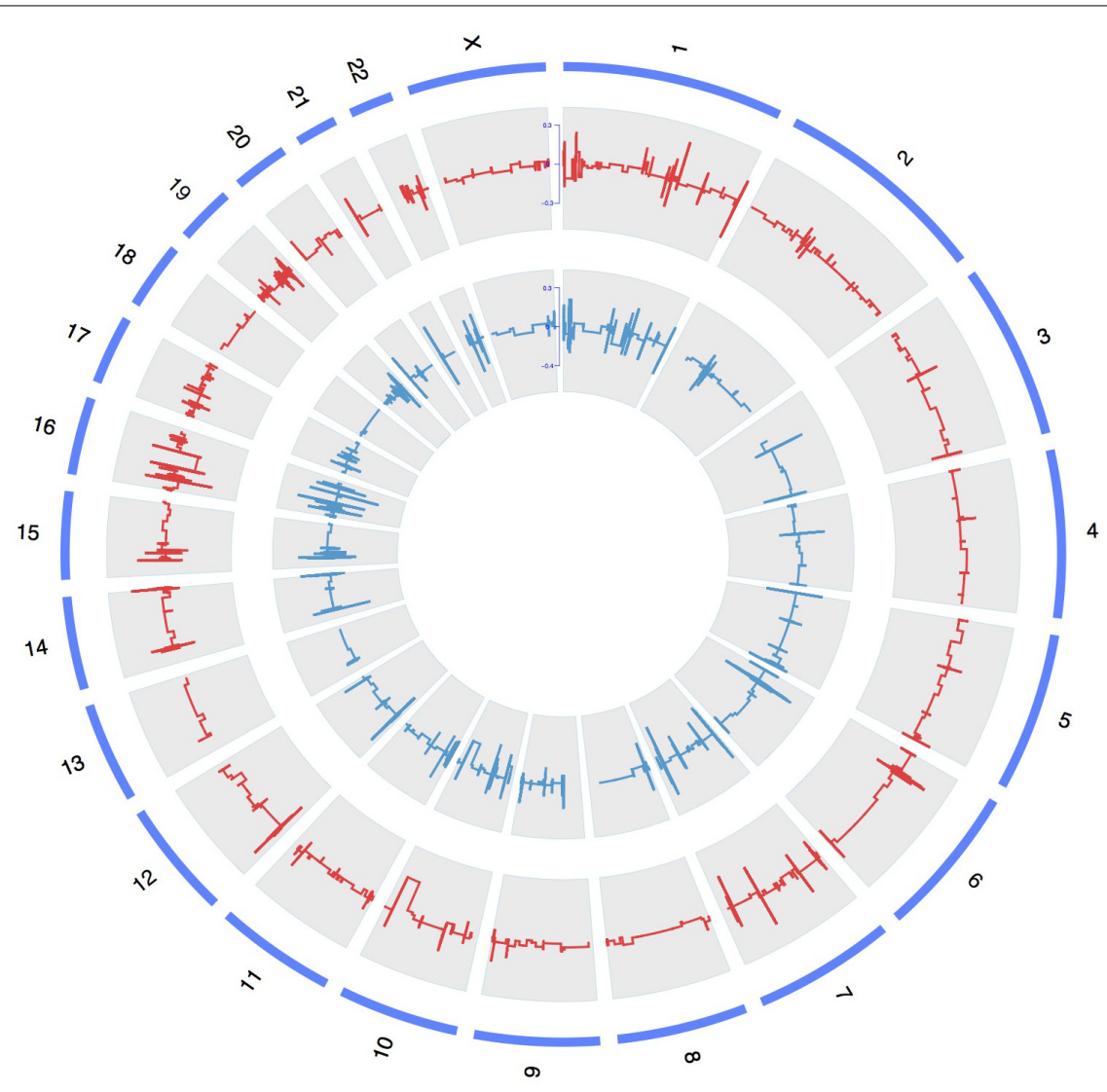

FIGURE 1 | A whole-genome view of copy number variations in case and control groups. Circos plot for variants visualization with broad horizontal area from chromosome level. The outer, middle, and inner tracks display the chromosomes, CNV frequency in case group, and CNV frequency in control group. The lines above or under zero represent gain or loss. 
TABLE 2 | Copy number variant (CNV) detected by Fisher' test.

\begin{tabular}{|c|c|c|c|c|}
\hline CNV & Chromosome & Gene & Case with CNV & $P$-value \\
\hline Duplication & Chr1 & PCSK7 & 9 & 0.00040 \\
\hline Duplication & Chr22 & RRP7A & 7 & 0.00241 \\
\hline Duplication & Chr22 & SERHL & 7 & 0.00241 \\
\hline Duplication & Chr7 & TARP & 13 & 0.00394 \\
\hline Deletion & Chr2 & TTN & 8 & 0.00584 \\
\hline Duplication & Chr22 & SERHL2 & 6 & 0.00584 \\
\hline Duplication & Chr1 & NBPF3 & 6 & 0.00584 \\
\hline Deletion & Chr1 & PCSK7 & 6 & 0.00584 \\
\hline Duplication & Chr17 & KRTAP9-6 & 7 & 0.01239 \\
\hline Deletion & Chr2 & TTN-AS1 & 5 & 0.01404 \\
\hline Duplication & Chr7 & MTRNR2L6 & 5 & 0.01404 \\
\hline Deletion & Chr20 & FER1L4 & 8 & 0.01818 \\
\hline Deletion & Chr1 & FLG2 & 15 & 0.02036 \\
\hline Deletion & Chr1 & FLG-AS1 & 15 & 0.02036 \\
\hline Deletion & Chr19 & ZNF844 & 9 & 0.02292 \\
\hline Deletion & Chr1 & CROCC & 6 & 0.02292 \\
\hline Deletion & Chr1 & OR2L5 & 6 & 0.02643 \\
\hline Deletion & Chr1 & SPATA21 & 4 & 0.03347 \\
\hline Deletion & Chr1 & RPTN & 4 & 0.03347 \\
\hline Deletion & Chr11 & AHNAK & 10 & 0.03347 \\
\hline Deletion & ChrX & DMD & 4 & 0.03347 \\
\hline Duplication & Chr3 & MUC4 & 4 & 0.03347 \\
\hline Duplication & Chr16 & STX4 & 4 & 0.03347 \\
\hline Deletion & Chr22 & GGT3P & 4 & 0.03347 \\
\hline Deletion & Chr1 & OR2L3 & 4 & 0.03347 \\
\hline Deletion & Chr19 & ZNF611 & 4 & 0.03347 \\
\hline Duplication & Chr10 & ANTXRL & 8 & 0.04273 \\
\hline
\end{tabular}

human embryonic hearts had a significantly higher level than other genes. Expression of PCSK7, RRP7A, and NBPF3 were also high just behind TNN.

\section{STRING Network Analysis}

We got 27 known pathogenic genes derived from the literature and publicly database. Then we used STRING database to explore the PPI network between CNV candidate genes and known pathogenic genes. Through PPI network, we found PCSK7 and TTN had more direct and obvious relation to known pathogenic genes (Figure 3). PCSK7 directly interacts with KDR and TTN indirectly interacts with $A N K R D 1$ and SGCD. These two genes could interact with other pathogenic genes via several other genes.

\section{DISCUSSION}

Total anomalous pulmonary venous connection is a rare congenital heart defect characterized by the misconnection of all four pulmonary veins, which could cause severe morbidity and mortality (Bando et al., 1996). Several genes have been associated TAPVC but the etiology of TAPVC is still complicated. To detect the underlying mechanism of TAPVC, we screened a cohort of 78 TAPVC cases and 100 healthy controls for rare CNVs and novel candidate genes, using whole exome sequencing (WES). Then we got seven totally novel candidate genes (PCSK7, RRP7A, SERHL, TARP, TTN, SERHL2, and NBPF3) that were associated with TAPVC. STRING network analysis demonstrated that PCSK7 and TTN which are highly related to known pathogenic genes, appear to play an important role in the genetic mechanism of TAPVC.

Both deletion and duplication of $\mathrm{CNV}$ could have been associated with congenital disorders (McLysaght et al., 2014). Recent data show that the frequency of duplications is approximately half of deletions and their phenotypes of heart malformation are much more diverse. It is possible that genomic deletions are more likely to cause dosage sensitivity compared with duplications because the fold change is greater for deletions.

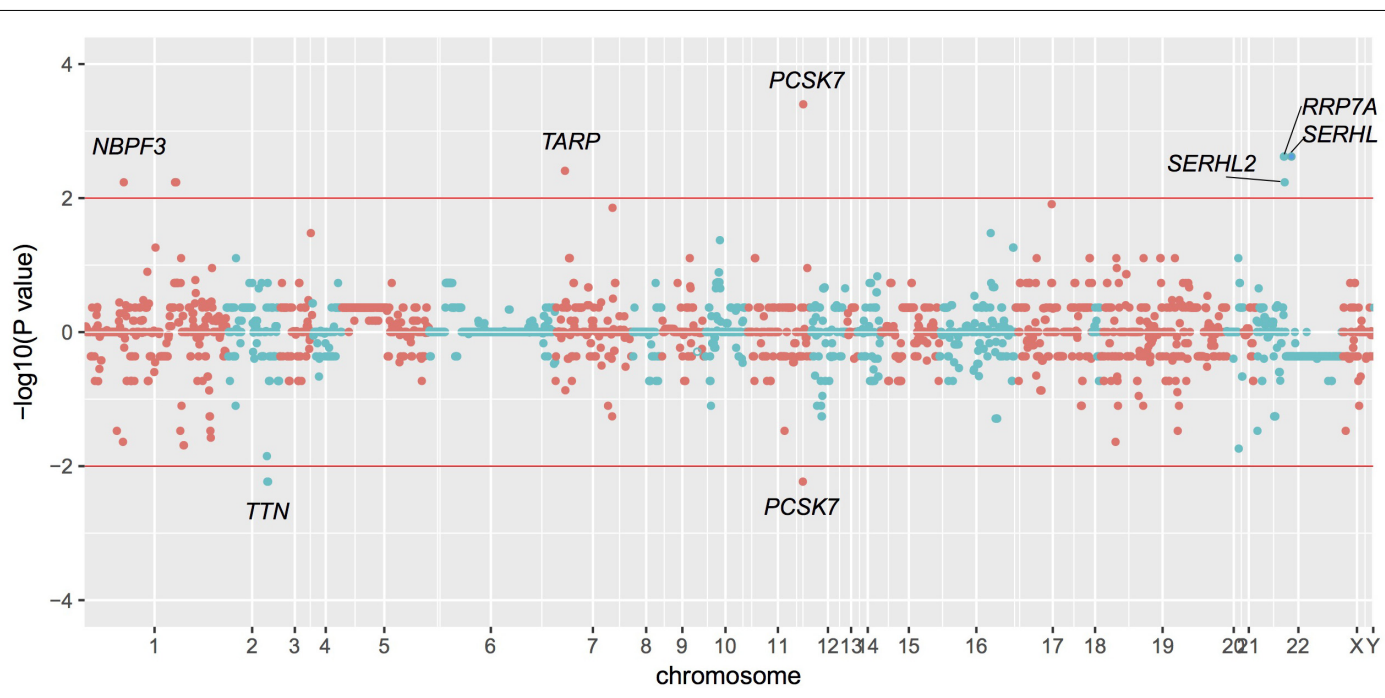

FIGURE 2 | Manhattan plot of CNVs detected by whole exome sequencing. CNVs detection by statistical comparisons between the case and control groups. Different colors of points mean CNVs from different chromosome. $P>0$ means duplication while $P<0$ means deletion. 
TABLE 3 | Expression patterns of candidate genes in human embryonic hearts at different time points.

\begin{tabular}{|c|c|c|c|c|c|c|c|c|}
\hline Chromosome & Gene & $\mathrm{S} 10$ & S11 & $\mathrm{S} 12$ & $\mathrm{~S} 13$ & S14 & $\mathrm{S} 15$ & S16 \\
\hline chr12 & GAPDH & 17.47 & 17.50 & 17.48 & 17.45 & 17.35 & 17.24 & 17.00 \\
\hline chr11 & PCSK7 & 7.01 & 7.04 & 7.05 & 7.07 & 7.18 & 7.17 & 7.05 \\
\hline chr22 & RRP7A & 7.00 & 6.93 & 7.03 & 6.69 & 7.05 & 6.93 & 6.88 \\
\hline chr22 & SERHL & 4.96 & 5.02 & 4.87 & 4.93 & 4.85 & 5.02 & 4.93 \\
\hline chr7 & TARP & 3.76 & 3.69 & 3.58 & 3.69 & 3.69 & 3.69 & 3.69 \\
\hline chr2 & TTN & 15.74 & 17.84 & 18.00 & 17.83 & 17.82 & 17.85 & 17.77 \\
\hline chr22 & SERHL2 & 5.09 & 5.10 & 5.07 & 5.10 & 5.10 & 5.10 & 5.10 \\
\hline chr1 & NBPF3 & 7.12 & 7.11 & 7.14 & 7.08 & 7.14 & 7.16 & 7.14 \\
\hline
\end{tabular}

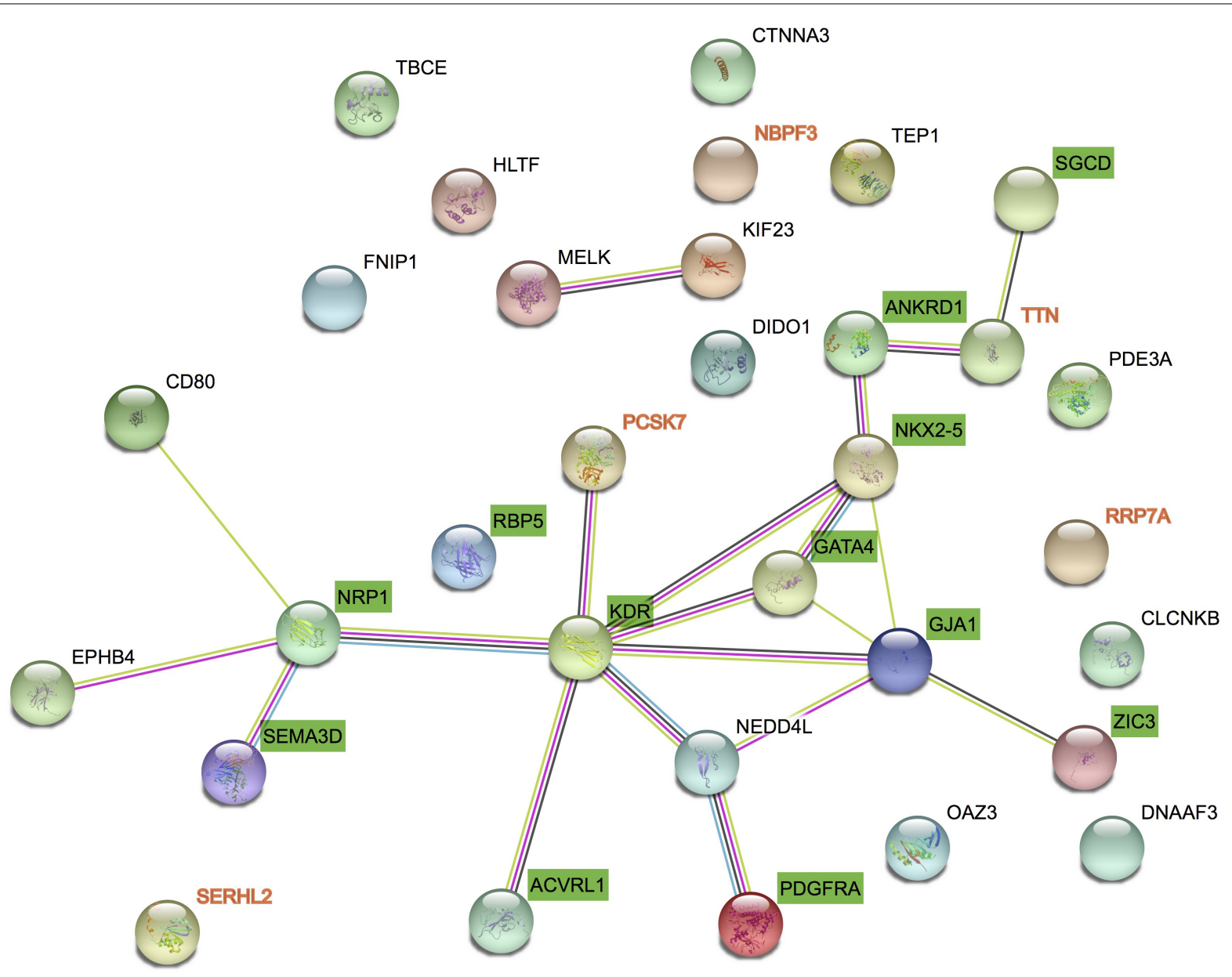

FIGURE 3 | Protein-protein interaction between candidate genes and known genes. STRING networks included 27 genes previously associated to TAPVC and 7 candidate genes highlighted by CNV detection analysis.

In seven candidate genes, we found deletion CNVs only in PCSK7 and TTN.

A total of $9(9 / 78,11.5 \%)$ patients had duplication and 6 $(6 / 78,7.7 \%)$ patients had deletion in PCSK7. PCSK7 (Proprotein convertase subtilisin/kexin type 7) is a member of the subtilisinlike proprotein convertase family (Constam et al., 1996). The genetic regulation of PCSK expression especially PCSK7 could bind to other genes to make huge impact on the blood pressure (Peloso et al., 2014; Turpeinen et al., 2015). Recent research of cardiovascular Disease (CVD) network using 1512 SNPs associated with 21 traits in genome-wide association showed PCSK7 connected closely to the incidence of CVD (Yao et al., $2015)$. In our study, $8(8 / 78,10.3 \%)$ patients were detected to have the deletion in TTN.TTN (Titin) encodes the sarcomere protein titin. Among its related pathways are dilated cardiomyopathy (DCM) and cardiac conduction (Hinson et al., 2015). A large literature suggested that majority of familial and sporadic DCM had the rare variants in TTN (Herman et al., 2012; 
Ware et al., 2016). A study found TTN and ANKRD1 which was an important pathogenic gene of TAPVC could combine to cause DCM (Arimura et al., 2009). And expression of TNN and PCSK7 were higher than other genes in human embryonic hearts. Above all, PCSK7 and TTN can be a totally novel candidate gene for TAPVC pathogenesis but the underlying mechanism remains unclear.

We found seven patients had duplications in SERHL, RRP7A and six patients in SERHL2. SERHL, SERHL2, and RRPTA, these genes are all located on chromosome 22q13. SERHL (Serine hydrolase-like) was encoded within the mRNA is an open reading frame of 311 amino acids which shows identity to a family of serine hydrolases (Sadusky et al., 2001). SERHL was found in tetralogy of Fallot patients and was associated with DNA methylation abnormalities (Serra-Juhe et al., 2015). SERHL2 also belongs to the serine hydrolase family, while its functional role is yet to be elucidated, and other nearby genes in the region, such as RRP7A, could also be biological candidates linked to 22q13 deletion syndrome (Okada et al., 2018). Patients with 22q13 duplication have been reported to have the clinical diagnosis of cardiovascular abnormalities and intrauterine growth restriction (Chen et al., 2003; Rahikkala et al., 2013). The relationship between RRP7A, SERHL, and SERHL2 and TAPVC needs to be further validated experimentally. Thus far, the functions of these genes in cardiovascular development remain unknown, and they be might newly associated with TAPVC pathogenesis.

In our research, TARP had the most patients than other genes, $13(13 / 78,16.7 \%)$ patients with duplication was detected in TARP.TARP (TCR gamma alternate reading frame protein) which is a marker for $\mathrm{T}$ cells and NKT cells and uniquely expressed in males in prostate epithelial cells and prostate cancer cells (Littman et al., 1987). It has been reported to be a biomarker for viral myocarditis (Rowe et al., 2018). We also found $6(6 / 78,7.7 \%)$ patients had duplication in NBPF3.NBPF3 (NBPF member 3) is a member of the neuroblastoma breakpoint

\section{REFERENCES}

Ammash, N. M., Seward, J. B., Warnes, C. A., Connolly, H. M., O’Leary, P. W., and Danielson, G. K. (1997). Partial anomalous pulmonary venous connection: diagnosis by transesophageal echocardiography. J. Am. Coll. Cardiol. 29, 1351-1358. doi: 10.1016/S0735-1097(97)82758-1

Arimura, T., Bos, J. M., Sato, A., Kubo, T., Okamoto, H., Nishi, H., et al. (2009). Cardiac ankyrin repeat protein gene (ANKRD1) mutations in hypertrophic cardiomyopathy. J. Am. Coll. Cardiol. 54, 334-342. doi: 10.1016/j.jacc.2008. 12.082

Bjornard, K., Riehle-Colarusso, T., Gilboa, S. M., and Correa, A. (2013). Patterns in the prevalence of congenital heart defects, metropolitan Atlanta, 1978 to 2005. Birth Defects Res. A Clin. Mol. Teratol. 97, 87-94. doi: 10.1002/bdra. 23111

Bando, K. O., Turrentine, M. W., Ensing, G. J., Sun, K., Sharp, T. G., Sekine, Y., et al. (1996). Surgical management of total anomalous pulmonary venous connection. Thirty-year trends. Circulation 94, II12-II16.

Bleyl, S. B., Botto, L. D., Carey, J. C., Young, L. T., Bamshad, M. J., Leppert, M. F., et al. (2006). Analysis of a Scottish founder effect narrows the TAPVR1 gene interval to chromosome 4q12. Am. J. Med. Genet. A 140, 2368-2373. doi: 10.1002/ajmg.a.31471 family (NBPF) which consists of dozens of recently duplicated genes primarily located in segmental duplications on human chromosome 1 (Vandepoele et al., 2005). NBPF3 is reported to express in a variety of tissues (Vandepoele and van Roy, 2007). Our study is flawed. First, the lack of parental samples limited our ability to study the genetic backgrounds of the variants. Second, we lack the information of prognosis of TAPVC cases. In addition, the functions of our candidate genes need to be further verified with fundamental research. In summary, an effective analytical bioinformatics strategy allowed us to identify CNVs in novel genes that play a vital role in TAPVC pathology. Based on the results of CNV discovery in a case-control cohort, our study found evidence that CNVs of seven candidate genes (PCSK7, RRP7A, SERHL, TARP, TTN, SERHL2, and NBPF3) could contribute to the genetic etiology of TAPVC. Our candidate genes open new fields of investigation into TAPVC pathology and provide novel insights into pulmonary vein development.

\section{AUTHOR CONTRIBUTIONS}

SC conceived and designed the project, responsible for the overall content and revised the manuscript. XS, LC, WL, JW, and XJ performed bioinformatics analysis of CNV data. BC, JS, YX, QF, and YaL collected the clinical data. ZL, GL, and YuL carried out all experiments. XS and LC prepared the manuscript. All authors have seen and approved the final manuscript.

\section{ACKNOWLEDGMENTS}

We would like to acknowledge funding from the National Natural Science Foundation of China $(81720108003,81670285$, and 81741066), a multi-center clinical research project of Shanghai Jiao Tong University (DLY201609), and Innovation Program of Shanghai Municipal Education Commission (15ZZ055).

Brohee, S., Faust, K., Lima-Mendez, G., Vanderstocken, G., and van Helden, J. (2008). Network analysis tools: from biological networks to clusters and pathways. Nat. Protoc. 3, 1616-1629. doi: 10.1038/nprot.2008.100

Burroughs, J. T., and Edwards, J. E. (1960). Total anomalous pulmonary venous connection. Am. Heart J. 59, 913-931. doi: 10.1016/0002-8703(60)90414-2

Chen, C. P., Chern, S. R., Chang, T. Y., Lee, C. C., Chen, L. F., Tzen, C. Y., et al. (2003). Prenatal diagnosis of mosaic ring chromosome 22 associated with cardiovascular abnormalities and intrauterine growth restriction. Prenat. Diagn. 23, 40-43. doi: 10.1002/pd.517

Constam, D. B., Calfon, M., and Robertson, E. J. (1996). SPC4, SPC6, and the novel protease SPC7 are coexpressed with bone morphogenetic proteins at distinct sites during embryogenesis. J. Cell Biol. 134, 181-191. doi: 10.1083/jcb.134. 1.181

De Las Rivas, J., and Fontanillo, C. (2010). Protein-protein interactions essentials: key concepts to building and analyzing interactome networks. PLoS Comput. Biol. 6:e1000807. doi: 10.1371/journal.pcbi.1000807

Glessner, J. T., Bick, A. G., Ito, K., Homsy, J., Rodriguez-Murillo, L., Fromer, M., et al. (2014). Increased frequency of de novo copy number variants in congenital heart disease by integrative analysis of single nucleotide polymorphism array and exome sequence data. Circ. Res. 115, 884-896. doi: 10.1161/CIRCRESAHA. 115.304458 
Greenway, S. C., Pereira, A. C., Lin, J. C., DePalma, S. R., Israel, S. J., Mesquita, S. M., et al. (2009). De novo copy number variants identify new genes and loci in isolated sporadic tetralogy of Fallot. Nat. Genet. 41, 931-935. doi: 10.1038/ ng. 415

Hanemaaijer, N. M., Sikkema-Raddatz, B., van der Vries, G., Dijkhuizen, T., Hordijk, R., van Essen, A. J., et al. (2012). Practical guidelines for interpreting copy number gains detected by high-resolution array in routine diagnostics. Eur. J. Hum. Genet. 20, 161-165. doi: 10.1038/ejhg.2011.174

Herman, D. S., Lam, L., Taylor, M. R., Wang, L., Teekakirikul, P., Christodoulou, D., et al. (2012). Truncations of titin causing dilated cardiomyopathy. N. Engl. J. Med. 366, 619-628. doi: 10.1056/NEJMoa1110186

Hinson, J. T., Chopra, A., Nafissi, N., Polacheck, W. J., Benson, C. C., Swist, S., et al. (2015). Heart Disease. Titin mutations in iPS cells define sarcomere insufficiency as a cause of dilated cardiomyopathy. Science 349, 982-986. doi: $10.1126 /$ science.aaa5458

Hitz, M. P., Lemieux-Perreault, L. P., Marshall, C., Feroz-Zada, Y., Davies, R., Yang, S. W., et al. (2012). Rare copy number variants contribute to congenital left-sided heart disease. PLoS Genet. 8:e1002903. doi: 10.1371/journal.pgen. 1002903

Li, J., Yang, S., Pu, Z., Pu, Z., Dai, J., Jiang, T., et al. (2017). Wholeexome sequencing identifies SGCD and ACVRL1 mutations associated with total anomalous pulmonary venous return (TAPVR) in Chinese population. Oncotarget 8, 27812-27819. doi: 10.18632/oncotarget.15434

Li, Y., Klena, N. T., Gabriel, G. C., Klena, N. T., Gabriel, G. C., Liu, X., et al. (2015). Global genetic analysis in mice unveils central role for cilia in congenital heart disease. Nature 521, 520-524. doi: 10.1038/nature14269

Littman, D. R., Newton, M., Crommie, D., Ang, S. L., Seidman, J. G., Gettner, S. N., et al. (1987). Characterization of an expressed CD3-associated Ti gammachain reveals C gamma domain polymorphism. Nature 326, 85-88. doi: 10. $1038 / 326085 \mathrm{a} 0$

McDowall, M. D., Scott, M. S., and Barton, G. J. (2009). PIPs: human proteinprotein interaction prediction database. Nucleic Acids Res. 37, D651-D656. doi: 10.1093/nar/gkn870

McLysaght, A., Makino, T., Grayton, H. M., Tropeano, M., Mitchell, K. J., Vassos, E., et al. (2014). Ohnologs are overrepresented in pathogenic copy number mutations. Proc. Natl. Acad. Sci. U.S.A. 111, 361-366. doi: 10.1073/ pnas. 1309324111

Mlynarski, E. E., Sheridan, M. B., Xie, M., Guo, T., Racedo, S. E., McDonaldMcGinn, D. M., et al. (2015). Copy-Number variation of the glucose transporter gene SLC2A3 and congenital heart defects in the 22q11.2 deletion Syndrome. Am. J. Hum. Genet. 96, 753-764. doi: 10.1016/j.ajhg.2015.03.007

Nash, D., Arrington, C. B., Kennedy, B. J., Yandell, M., Wu, W., Zhang, W., et al. (2015). Shared segment analysis and next-generation sequencing implicates the retinoic acid signaling pathway in Total Anomalous Pulmonary Venous Return (TAPVR). PLoS One 10:e0131514. doi: 10.1371/journal.pone.0131514

Nolan, T., Hands, R. E., and Bustin, S. A. (2006). Quantification of mRNA using real-time RT-PCR. Nat. Protoc. 1, 1559-1582. doi: 10.1038/nprot.2006.236

Okada, Y., Momozawa, Y., Sakaue, S., Kanai, M., Ishigaki, K., Akiyama, M., et al. (2018). Deep whole-genome sequencing reveals recent selection signatures linked to evolution and disease risk of Japanese. Nat. Commun. 9:1631. doi: 10.1038/s41467-018-03274-0

Peloso, G. M., Auer, P. L., Bis, J. C., Voorman, A., Morrison, A. C., Stitziel, N. O., et al. (2014). Association of low-frequency and rare coding-sequence variants with blood lipids and coronary heart disease in 56,000 whites and blacks. Am. J. Hum. Genet. 94, 223-232. doi: 10.1016/j.ajhg.2014.01.009

Pinto, D., Darvishi, K., Shi, X., Rajan, D., Rigler, D., Fitzgerald, T., et al. (2011). Comprehensive assessment of array-based platforms and calling algorithms for detection of copy number variants. Nat. Biotechnol. 29, 512-520. doi: 10.1038/ nbt. 1852

Rahikkala, E., Forsstrom, L. M., Kokkonen, H., Knuutila, S., Mustonen, A., and Ignatius, J. (2013). Report of interstitial 22q13.1q13.2 microduplication in two siblings with distinctive dysmorphic features, heart defect and mental retardation. Eur. J. Med. Genet. 56, 389-396. doi: 10.1016/j.ejmg.2013. 05.004

Rowe, J. H., Delmonte, O. M., Keles, S., Stadinski, B. D., Dobbs, A. K., Henderson, L. A., et al. (2018). Patients with CD3G mutations reveal a role for human CD3gamma in Treg diversity and suppressive function. Blood 131, 2335-2344. doi: 10.1182/blood-2018-02-835561

Sadusky, T. J., Kemp, T. J., Simon, M., Carey, N., and Coulton, G. R. (2001). Identification of Serhl, a new member of the serine hydrolase family induced by passive stretch of skeletal muscle in vivo. Genomics 73, 38-49. doi: 10.1006/ geno. 2000.6483

Serra-Juhe, C., Cusco, I., Homs, A., Flores, R., Toran, N., and Perez-Jurado, L. A. (2015). DNA methylation abnormalities in congenital heart disease. Epigenetics 10, 167-177. doi: 10.1080/15592294.2014.998536

Southard, A. E., Edelmann, L. J., and Gelb, B. D. (2012). Role of copy number variants in structural birth defects. Pediatrics 129, 755-763. doi: 10.1542/peds. 2011-2337

Thummar, A. C., Phadke, M. S., Lanjewar, C. P., Nabar, A. A., and Kerkar, P. G. (2014). Supracardiac total anomalous pulmonary venous drainage with giant superior vena cava aneurysm: a rare combination. J. Am. Coll. Cardiol. 63:e51. doi: 10.1016/j.jacc.2013.11.071

Turpeinen, H., Seppala, I., Lyytikainen, L. P., Raitoharju, E., Hutri-Kähönen, N., Levula, M., et al. (2015). A genome-wide expression quantitative trait loci analysis of proprotein convertase subtilisin/kexin enzymes identifies a novel regulatory gene variant for FURIN expression and blood pressure. Hum. Genet. 134, 627-636. doi: 10.1007/s00439-015-1546-5

Vandepoele, K., and van Roy, F. (2007). Insertion of an HERV(K) LTR in the intron of NBPF3 is not required for its transcriptional activity. Virology 362, 1-5. doi: 10.1016/j.virol.2007.01.044

Vandepoele, K., Van Roy, N., Staes, K., Speleman, F., and van Roy, F. (2005). A novel gene family NBPF: intricate structure generated by gene duplications during primate evolution. Mol. Biol. Evol. 22, 2265-2274. doi: 10.1093/molbev/ msi222

Ware, J. S., Li, J., Mazaika, E., Yasso, C. M., DeSouza, T., Thomas, P. C., et al. (2016). Shared genetic predisposition in peripartum and dilated cardiomyopathies. N. Engl. J. Med. 374, 233-241. doi: 10.1056/NEJMoa150 5517

Wellcome Trust Case Control Consortium., Craddock, N., Hurles, M. E., Pearson, R. D., Plagnol, V., Robson, S., et al. (2010). Genome-wide association study of CNVs in 16,000 cases of eight common diseases and 3,000 shared controls. Nature 464, 713-720. doi: 10.1038/nature08979

Yao, C., Chen, B. H., Joehanes, R., Otlu, B., Zhang, X., Liu, C., et al. (2015). Integromic analysis of genetic variation and gene expression identifies networks for cardiovascular disease phenotypes. Circulation 131, 536-549. doi: 10.1161/ CIRCULATIONAHA.114.010696

Conflict of Interest Statement: The authors declare that the research was conducted in the absence of any commercial or financial relationships that could be construed as a potential conflict of interest.

The reviewer DZ declared a shared affiliation, with no collaboration, with several of the authors, XS, LC, XJ, BC, WL, JW, GL, YX, JS, QF, YaL, and SC, to the handling Editor at the time of review.

Copyright (C) 2018 Shi, Cheng, Jiao, Chen, Li, Liang, Liu, Wang, Liu, Xu, Sun, Fu, Lu and Chen. This is an open-access article distributed under the terms of the Creative Commons Attribution License (CC BY). The use, distribution or reproduction in other forums is permitted, provided the original author(s) and the copyright owner(s) are credited and that the original publication in this journal is cited, in accordance with accepted academic practice. No use, distribution or reproduction is permitted which does not comply with these terms. 